\title{
Effect of prostate cancer cell line supernatant on functional polarization in macrophages
}

\author{
Mazalova L ${ }^{1}$, Sladek $Z^{1}$, Raudenska $\mathrm{M}^{2}$, Balvan $\mathrm{J}^{2}$, Gumulec $\mathrm{J}^{2}$, Masarik $\mathrm{M}^{2}$ \\ Mendel University in Brno, Faculty of Agronomy, Brno, Czech Republic. zbysek.sladek@mendelu.cz
}

\begin{abstract}
OBJECTIVES: We aimed on effect of supernatant derived from prostate cancer cell line PC-3 on M1/M2 functional polarization in macrophages.

BACKGROUND: Cytokines play an important role in carcinogenesis. Most of them are produced by macrophages. Macrophages are divided into groups M1 or M2. Classical phenotype macrophages M1 support proinflammatory effects and produce pro-inflammatory cytokines, such as interleukin 6 (IL-6), interleukin 12 (IL-12), tumor necrosis factor $\alpha$ (TNF- $\alpha$ ). Macrophages exhibiting a phenotype M2 secrete anti-inflammatory cytokines, e. g. interleukin 10 (IL-10), transforming growth factor $\beta$ (TGF- $\beta$ ).

METHODS: Peripheral blood monocytes were cultivated for 7 days and during this time went through a differentiation into macrophages. Macrophages were stimulated for 24 hours by lipopolysaccharide (LPS) as a positive control and cultivated with supernatant for another 24 hours.

RESULTS: Macrophages cultivated without LPS and without supernatant were used as negative control. Relative expression of IL-6, IL-10, IL-12 and TNF- $\alpha$ was measured by Quantitative real-time PCR. Expression of pro-inflammatory cytokines was lower in macrophages with supernatant compared to positive control.

CONCLUSION: Expression of pro-inflammatory cytokines was lower in macrophages with supernatant (MФ+sup) compared to positive control (MФ+LPS). Effect of the supernatant on expression of IL-6, IL-10, IL-12 and TNF- $\alpha$ was not confirmed (Tab. 1, Fig. 5, Ref. 15). Text in PDF www.elis.sk.

KEY WORDS: prostate, cancer, supernatant, macrophages, cytokines.
\end{abstract}

\section{Introduction}

Prostate cancer is the most commonly diagnosed non-cutaneous cancer and the second leading cancer-related cause of death for men. In 2016 over 200,000 new cases of prostate cancer were diagnosed in the United States (1). Approximately 307,000 deaths were recorded globally in 2012. Incidence rates were higher in Western and Northern Europe, North America and Oceania (2). Cancer is a complex process and cytokines play an important role in its development. Pro-inflammatory action of cytokines (IL-1, TNF- $\alpha$, IL-6) are involved in prostate cancer development (3). These cytokines stimulate cell proliferation and reduce apoptosis, they are involved in angiogenesis and formation of metastasis. An increase of anti-inflammatory cytokines should limit the risk of cancer and reduce activation of signalling pathways (4). It is now understood that tumor cells interact with cells of the surrounding

${ }^{1}$ Mendel University in Brno, Faculty of Agronomy, Brno, Czech Republic, and ${ }^{2}$ Masaryk University, Faculty of Medicine, Brno, Czech Republic

Address for correspondence: Z. Sládek, Prof., MVD, PhD, Department of Morphology, Physiology and Animal Genetics, Faculty of Agronomy, Mendel University in Brno, Zemedelska 1, CZ-613 00 Brno, Czech Republic. Phone: +420.545133151

Acknowledgement: The research was financially supported by the IGA AF MENDELU IP 27/2015. stroma and drive tumor progression. The tumor microenvironment is a chronic site of inflammation (2). The cancer microenvironment is known to comprise of tumor and stromal cells and involves a complex network of reciprocal interaction between tumor cells and inflammatory cells (1).

The tumor-associated macrophages (TAMs) are highly active immune effectors cells that may either positively or negatively regulate the division of various malignant cells, depending on the biological context. TAMs represent the main population of leukocytes, which occurs in many tumors, and their exact role is not yet fully understood (5). TAMs are significantly involved in the progression of tumors. They promote the formation of metastases, angiogenesis (secrete angiogenic factors), tumor growth (producing growth hormones), enhance the migration and invasiveness of tumor cells (6). This effect can be understood via the functional plasticity of macrophages, which can be classified as M1 or M2 (1). Macrophages are recruited to tumors by growth factors and chemokines, which are often produced by the cancer cells and stroma cells in tumor. The potential angiogenesis factors secreted by TAMs include cytokines, such as IL- 8 and TNF- $\alpha$. It was reported that coculture of macrophages with breast cancer cell stimulated the macrophages and up-regulated production of TNF- $\alpha$; subsequently enhanced cancer cell invasion. On the other hand, the possible anti-tumor function of TAMs may be realized through IL-12 expression. The cytokines profile of microenvi- 
ronment and localization of TAMs may influence the function of TAMs (6).

Macrophages can change repeatedly functional profile M1 or M2 in response to changes occurring in their surroundings and the proces is called switch off. According to the stimulus macrophages are divided into M1 and M2 due to their functional polarization. Macrophages differentiate into subpopulations of cells that express specific sets of cell surface molecules and secrete specific combination of cytokines and chemokines. Classical phenotype of macrophages M1 support pro-inflammatory effects and produce pro-inflammatory cytokines (IL-6, IL-12, TNF- $\alpha$ ), while macrophages exhibiting a phenotype $\mathrm{M} 2$ secrete anti-inflammatory cytokines (IL-10, TGF- $\beta$ ) (1). The plastic nature of macrophages allows to re-polarize them into a more tumoricidal phenotype. Reversion of the tumor-associated M2-like status into a M1-like phenotype has been shown to improve anti-tumor responses. Interaction of already polarized M2-like macrophages with Th1 cells results in a repolarization of M2-like into M1-like macrophages with induction of costimulatory molecules and production of IL12 (7). Due to the anti-cancer immune response M1 macrophages are considered as beneficial, while M2 likely promote tumor progression (5). Depletion of M2 macrophages results in 1) decrease of tumor growth in various murine cancer models, 2) reduction of tumor volume and reduction of blood vessel density, 3) increase response to chemotherapy, and 4) reduction of macrophages infiltration with reduction of bone metastasis (7).

Can it be expected that prostatic cancer cells secrete factors, which modulate phenotypic manifestations in macrophages? Modulation of the macrophages immunophenotype from M1 into M2 by the microenvironment is not well understood (1). In this study we aimed on how supernatant from prostate cancer cell line PC-3 effects functional polarization in macrophages. The aim was to discover the basic interaction between macrophage-supernatant in the expression of selected cytokines. We investigated the expression of cytokines TNF- $\alpha$, IL-6, IL-10 and IL-12. These four cytokines were selected because of their typical representation in M1 or M2 phenotype.

\section{Materials and methods}

\section{Human rights}

This study has been carried out in accordance with The Code of Ethics of the World Medical Association (Declaration of Helsinki) for experiments involving humans. Informed consent was obtained for experimentation with human subjects and the institution's ethics committee approved the study. The privacy rights of human subjects were always observed.

\section{PC-3 cell line}

The PC-3 cell line is derived from the 4th grade of prostatic adenocarcinoma, androgen independent (HPA Culture Collections, Salisbury, UK).

\section{Cultivation of monocytes}

Human monocytes, as part of PBMC (Peripheral Blood Mononuclear Cell) were isolated from $40 \mathrm{ml}$ male blood by Histopaque
1077 (Sigma-Aldrich, St. Louis, USA) protocol. The samples came from 10 healthy men. Blood was diluted in a ratio $1: 1$ by PBS (Dulbecco's Phosphate Buffered Saline, without $\mathrm{Ca}$ and $\mathrm{Mg}$, Lonza Group Ltd, Basel Switzerland) and centrifugated (speed $2000 \mathrm{rpm}$, time $40 \mathrm{~min}$, brake 0, temp. $24-40{ }^{\circ} \mathrm{C}$ ). Than PBMC were obtained by aspiration from the respective ring of density gradient from Histopaque 1077 and centrifugated with PBS (speed $1500 \mathrm{rpm}$, time $10 \mathrm{~min}$, brake 9, temp. $21-29^{\circ} \mathrm{C}$ ). Cells in pellet were suspended in RPMI 1640 with L-glutamine (Lonza Group Ltd, Basel Switzerland) and seeded into Multi well Culture Plate in concentration $10^{6} /$ well. Cultivation was carried out for 7 days with GM-CSF (Recombinant Human Granulocyte Macrophage ColonyStimulating Factor, Animal Origin Free, Gibco, Thermo Fisher Scientific, Massachusetts, USA), preparation: $20 \mu \mathrm{g}$ GM-CSF + $1 \mathrm{ml} \mathrm{DPBS}+1 \mu 10.1 \% \mathrm{BSA}$ ) in concentration $5 \mu \mathrm{l}$ of prepared GM-CSF/1 ml cell culture medium RPMI 1640 with L-glutamin in $5 \% \mathrm{CO}_{2}$ at $37^{\circ} \mathrm{C}$. On day 7 , macrophages were stimulated by LPS (from E. Coli, Sigma-Aldrich, St. Louis, USA) preparation: $2.5 \mathrm{mg}$ LPS $+1 \mathrm{ml}$ PBS) in concentration $20 \mu \mathrm{l}$ of prepared LPS/ 1 $\mathrm{ml}$ cell culture medium and with PC-3 prostate cancer cell line supernatant. LPS was elected as a pro-inflammatory indicator due to the expected pro-inflammatory effect of supernatant. Macrophages stimulated only by LPS served as a positive control. Macrophages without LPS and without supernatant served as a negative control. Supernatant was obtained 5 days after starting of cultivation of cancer cells. Samples were divided into 4 variants: macrophages only (as a negative control) - designation $\mathrm{M} \Phi$; macrophages + LPS (as a positive control) - designation M $\Phi+\mathrm{LPS}$; macrophages + supernatant - designation MФ+sup; macrophages + supernatant + LPS - designation M $\Phi+$ sup+LPS. After 24 hours macrophages were harvested mechanically by repeated washes in medium RPMI 1640. All processes (centrifugation, aspiration) associated with handling with the supernatant were carried out prior to in vitro cultivation with macrophages.

\section{RNA Isolation and Reverse Transcription}

TriPure Isolation Reagent (Roche, Basel, Switzerland) was used for RNA isolation. The isolated RNA used for cDNA synthesis. RNA (1000 ng) was transcribed using transcriptor first strand cDNA synthesis kit (Roche, Basel, Switzerland), which was applied according to manufacturer's instructions. The cDNA (20 $\mu$ l) prepared from the total RNA was diluted with RNase free water to $100 \mu \mathrm{l}$ and the amount of $5 \mu \mathrm{l}$ was directly analysed by using the LightCycler®480 II System (Roche, Basel, Switzerland).

\section{Quantitative real-time polymerase chain reaction}

qRT-PCR was performed using the TaqMan gene expression assays with the LightCycler®480 II System (Roche, Basel, Switzerland) and the amplified DNA was analysed by the comparative $\mathrm{Ct}$ method using $\beta$-actin as an endogenous control. The primer and probe sets for ACTB (assay ID: $\beta$-actin (Hs_99999903), TNF (Hs01113624_g1), IL-6(Hs00174131_m1), IL-6R (Hs01075666_ m1), IL-10 (Hs00961622_m1), IL-10RB (Hs00175123_m1) and IL-12A (Hs00168405_m1), were selected from TaqMan gene expression assays (Thermo Fisher Scientific, Massachusetts, USA). 
qRT-PCR was performed under the following amplification conditions: total volume of $20 \mu \mathrm{l}(5 \mu \mathrm{l} \mathrm{cDNA}+0.8 \mu \mathrm{l}$ probe $+8.2 \mu \mathrm{l}$ master mix $+6 \mu$ water), initial incubation at $50{ }^{\circ} \mathrm{C} / 2 \mathrm{~min}$ followed by denaturation at $95^{\circ} \mathrm{C} / 10 \mathrm{~min}$, then 45 cycles at $95^{\circ} \mathrm{C} / 15$ $\mathrm{s}$ and at $60^{\circ} \mathrm{C} / 1 \mathrm{~min}$.

\section{Statistics}

Statistics and $\mathrm{p}$ values for cytokine expression were computed by software Statistica 12 (StatSoft, Inc., a part of Dell, Tulsa, USA) by using Factorial ANOVA, Fischer post hoc test and Correlation analysis. Statistical significance was declared when $\mathrm{p}$ value was equal to or less than 0.05 .

\section{Results and discussion}

The aim was to discover the basic interaction between macrophage-supernatant in the expression of selected cytokines. From the literature it is known that cancer cells in vitro release substances influencing the expression of genes. The interaction between cancer cells and TAMs may improve the processes leading to the development of cancer. For example, the interaction may enhance cancer cell growth, invasion, metastasis, angiogenesis by stimulating TAMs or cancer cells to express multiple gene products that are involved in these processes (platelet-derived growth factor, colony-stimulating factor-1, intercellular adhesion molecule- 1 etc.) $(6,7)$. We chose the 24 hours experiment because of sufficient time for incubation of macrophages with the supernatant and also we used proven methodology in accordance with studies by Sánches-Reyes et al (2014) and Caras et al (2011). It is also sufficient time for the effect of LPS on macrophage activation into pro-inflammatory profile to obtain the positive control $(5,8)$.

In our study we included also the time of measurement 5 hours after starting of incubation of macrophages with supernatant in order to capture an earlier time of cytokines expression. LPS was selected as a pro-inflammatory indicator because of the expected anti-inflammatory effect of PC-3 supernatant. Therefore LPS serves as a positive control.

For analysis of polarization of macrophages we chose proinflammatory cytokines TNF- $\alpha$, IL- 12 , anti-inflammatory cytokine IL-10 and cytokine IL-6, which exhibits dual function. Measurements of the expression of cytokines were done at 5 and 24 hours

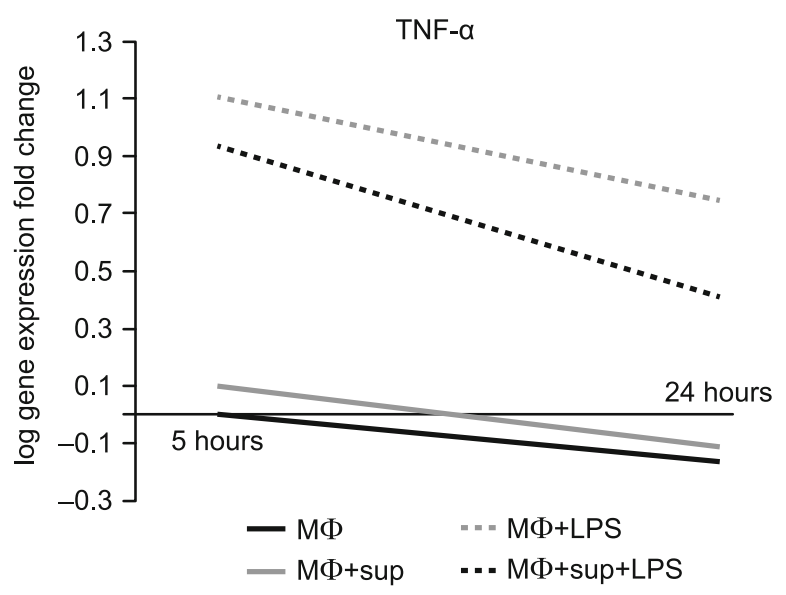

Fig. 1. Effect of supernatant and LPS on relative expression of TNF- $\alpha$.

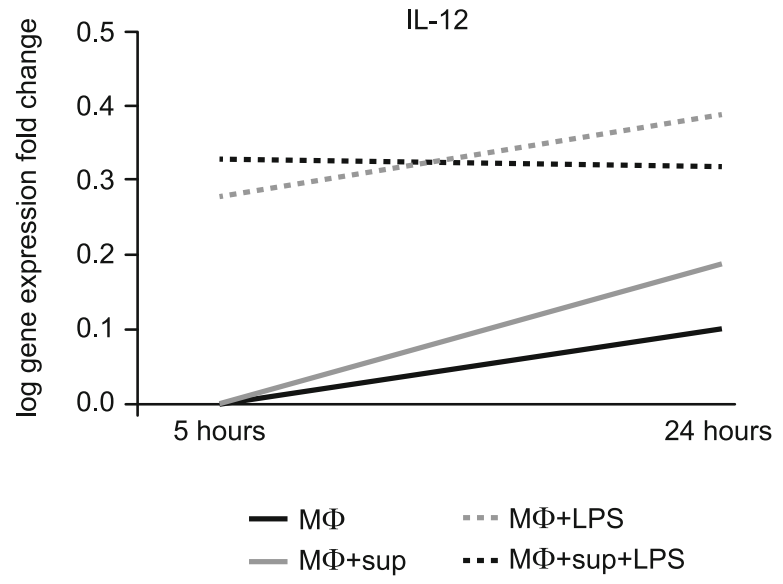

Fig. 2. Effect of supernatant and LPS on relative expression of IL-12.

after the start of cultivation, because of the effect of the LPS stimulation on genes with early and late response (9).

By multivariate test it was shown that a statistical significant effect had exposure time ( $\mathrm{p}$ value $=0.0000001)$ and presence of LPS $(p=0.0000001)$ in all samples. Further, the combination of time and LPS was also significant $(\mathrm{p}=0.016)$. Macrophages after LPS stimulation produced high levels of IL-12, IL- 6 and TNF- $\alpha$

Tab. 1. Significant $p$ values for expression of cytokines.

\begin{tabular}{|c|c|c|c|}
\hline Cytokine & Time & Samples & $\mathrm{p}$ \\
\hline \multirow[t]{3}{*}{ TNF- $\alpha$} & 5 hours & MФ (negative control) / MФ+LPS (positive control) & 0.000003 \\
\hline & 5 hours & $\mathrm{M} \Phi+\sup / \mathrm{M} \Phi+\sup +\mathrm{LPS}$ & 0.00004 \\
\hline & 24 hours & MФ (negative control) / MФ+LPS (positive control) & 0.000048 \\
\hline \multirow[t]{4}{*}{$\overline{\mathrm{IL}-12}$} & 5 hours & $\mathrm{M} \Phi+\sup / \mathrm{M} \Phi+\sup +$ LPS 0.0155 & \\
\hline & 5 hours & MФ (negative control) / M + +sup + LPS & 0.0181 \\
\hline & 24 hours & MФ (negative control) / MФ+LPS (positive control) & 0.0423 \\
\hline & 24 hours & MФ (negative control) / M $\Phi+$ sup+LPS & 0.0003 \\
\hline \multirow[t]{2}{*}{ IL-10 } & 24 hours & MФ+sup / MФ+LPS (positive control) & 0.0000001 \\
\hline & 5 hours & MФ (negative control) / M $\Phi+$ sup + LPS & 0.0368 \\
\hline IL-10R & 24 hours & MФ+sup / MФ+LPS (positive control) & 0.0059 \\
\hline
\end{tabular}




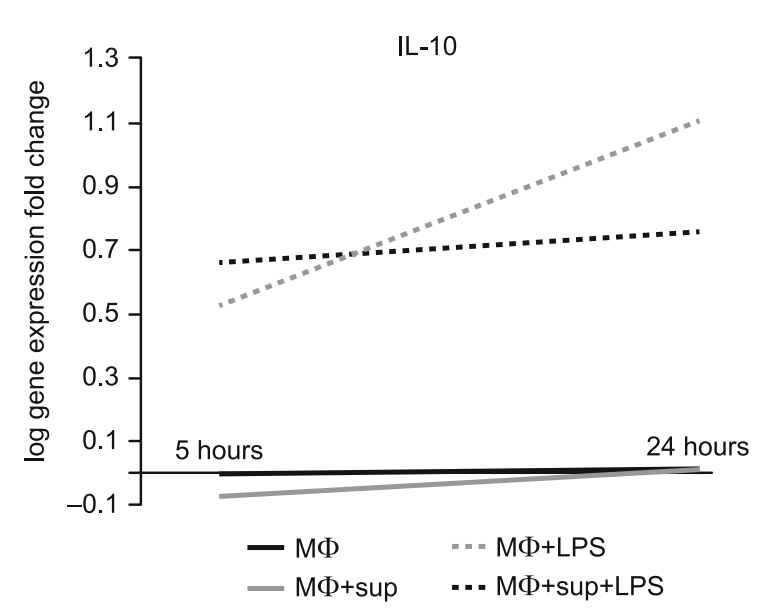

Fig. 3. Effect of supernatant and LPS on relative expression of IL-10.

in our experiment as expected. This is a well known fact, so macrophages stimulated by LPS were chosen as a positive control. Macrophages without LPS stimulus produced high levels of IL-10. Our results mentioned above are consistent with Caras et al (5). Effects of the supernatant were not statistically significant ( $\mathrm{p}$ value $=0.091)$ nor in combination with time $(\mathrm{p}=0.745)$ or in combination with LPS $(p=0.225)$. Time, LPS and the supernatant had no effect on the cytokine production $(\mathrm{p}=0.937)$.

As is evident from the results in Figure 1, in samples M $\Phi+$ sup was shown a decreasing expression of TNF- $\alpha$ compared to postitive control M $\Phi+\mathrm{LPS}$. However, the supernatant did not have a statistically significant role. Very similar results-including statistical significance and LPS stimulation of macrophages-were published by Sánchez-Reyes et al (2014). However, the authors stated that presence of supernatant from cancer cells prevented secretion of TNF- $\alpha$. It is necessary to mention that this study was conducted on cervical cancer cell supernatant (8). In our study supernatant derived from prostate cancer cell line PC-3 did not affect production of TNF- $\alpha$. Macrophages have an ability to recognize pathogens, such as bacteria E. coli containing LPS, from the outside environment through pathogen-associated molecular patterns. LPS represent an important endotoxin to which the immune system reacts by strong response (10). Bacterial LPS causes important changes in gene expression in macrophages by altering histone deacetylase expression (9). LPS causes switch off in macrophages and induces them to M1 phenotype which is characterized by their ability to release pro-inflammatory cytokines, for example TNF- $\alpha$, IL-1 $\beta$, IL-12, IL-23 (8).

It could be expected that the production of pro-inflammatory cytokines will be higher in macrophages activated by LPS. M1 macrophages activated by microbial stimuli, such as LPS, produce high levels of TNF- $\alpha$ (5). LPS acts on different genes. The one group-early response genes which includes many pro-inflammatory cytokines-is induced transiently, peaking at 2 to 4 hours and repressed progressively from that time. Other genes are induced at later time points, up to 24 hours after stimulation. Repression of the earlier response genes is partly irreversible and it has been associated with the LPS tolerance (9). In this experiment both time intervals were measured. Statistically significant difference was shown in expression of TNF- $\alpha$ and these data are shown in Table 1. Therefore, LPS significantly increased expression at 5 and also at 24 hours. Nevertheless, at 24 hours after LPS stimulation, expression of TNF- $\alpha$ was lower than at 5 hours. The expression decreased over time. The results show that the PC-3 supernatant, unlike LPS, did not effect TNF- $\alpha$ expression.

IL-12 was the next pro-inflammatory cytokine measured in this study (Fig. 2). Results indicated that the relative expression of IL-12 in the samples $M \Phi+$ sup was lower than in the samples M $\Phi+$ LPS. IL-12 is a pro-inflammatory cytokine and therefore these results were expected. At 5 hours LPS had a significant effect only when it was combined with the supernatant. This means only in samples M $\Phi+$ sup+LPS. LPS alone was enough for significantly increasing the expression at 24 hours. Significant $p$ values are listed in Table 1. IL-12 is produced by macrophages in response to microbial pathogens (11). In measuring time 5 hours there was an immediate stimulation with LPS, which has microbial origin. Therefore, production of IL-12 by macrophages was higher. It is evident that supernatant derived from prostate cancer cell line PC-3 did not affected production of IL-12. This is in accordance with the study of Sánchez-Reyes et al (2014).

IL-10 was another cytokine detected in our study. This anti-inflammatory cytokine is produced by almost all immune cells, including monocytes and macrophages and tumor cells (4). In this case, LPS plays a major role in its expression as it is evident from Figure 3. LPS and supernatant were both needed for significant decrease of IL-10 expression at 5 hours. Supernatant decreases expression steadily. There is no reason for the phenotypic switching of macrophages by cancer cells and this result was expected (12). Macrophages were focused on production of pro-inflammatory cytokines in the presence of supernatant and similar results were published by Caras et al (2011). Effect of the only supernatant on anti-inflammatory IL-10 cytokine production was not statistically confirmed.

In the positive control (M $\Phi+\mathrm{LPS}$ ) expression of IL-10 significatly increased at 5 and also at 24 hours. Many cells of the

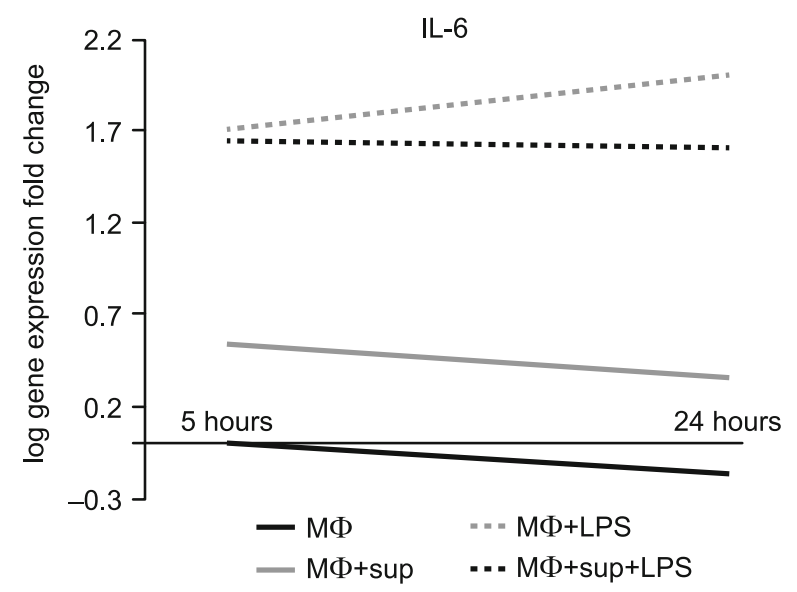

Fig. 4. Effect of supernatant and LPS on relative expression of IL-6. 


\section{6-521}

immune system were subjected to the action of cancer cells with deleterious or beneficial effect. It depends on the profile of substances secreted into the tumor microenvironment. For example, the substances secreted from cholangiocarcinoma cells exhibited M2 polarization of macrophages and also overproduction of cytokines including IL-10 (4). These results are in agreement with the above, after LPS stimulation the macrophages reached the phenotype M1. Increased expression of IL-10 in measuring time 24 hours after LPS stimulation can be explained as a tolerance to LPS (9). $P$ values for effect of supernatant and LPS on relative expression of IL-10 and IL-10 receptor are listed in Table 1.

The results of relative expression of IL- 6 are listed in Figure 4. IL-6 may play a role of a pro-inflammatory and also anti-inflammatory cytokine. Relative expressions of IL-6 were very similar to IL-10. Supernatant, LPS or time have no significant effect on IL-6 expression. In other studies it has been shown that IL-6 stimulates development of many tumors, such as melanoma, renal cell carcinoma, ovarian carcinoma, lymphoma breast carcinoma and also prostate carcinoma (2). IL-6 contributes to the development of tumor by promoting conversation of noncancer cells into tumor stem cells. On the other hand, IL-6 could be proposed as a therapeutic target in cancer (4). As a treatment of chronic inflammatory diseases the therapeutic blockade of IL-6 is offered. In view of the complex biology of IL-6, long-term global blockade of this cytokine should be carefully considered (13). The inhibition of IL-6 production and IL- 6 signalling suppresses the growth of colon cancer (14). IL-6 is secreted by both normal and neoplastic prostatic epithelial cells and can act as a growth factor for normal prostatic epithelial cells as well as for prostate cancer cells. Commonly used prostate cancer cell line PC-3 express high-affinity receptors for IL-6 and also secrete IL-6. Our results of relative expression of IL- 6 by macrophages are fully in line with the results of other studies (15).

Solis-Martínez et al (2018) published the study with results of experiments, in which they also cultivated the macrophages with PC-3 supernatant. These experiments strongly suggest the importance of the microenvironment induced by the PC-3 supernatant in the induction of the $\mathrm{M} 2$ profile. When the macrophages were incubated with PC-3 supernatant, the M1 markers practically disappeared in M1-induced groups of macrophages. Further when the macrophages were cultured with PC-3 supernatant, the concentration of growth factors (TNF- $\alpha$, GM-CSF, G-CSF etc.) was between 15 an $97 \mathrm{pg} / \mathrm{mL}$. When the growth factors were measured in the macrophage control group without any stimulus, secretion of growth factors was minimal (3-5 pg/mL) (1).

Figure 5 shows the correlation among cytokines. It is likely that cytokines interact with each other. Correlation processes were detected among the TNF / IL-6, IL-6R / IL-10R, IL-6 / IL-10. As
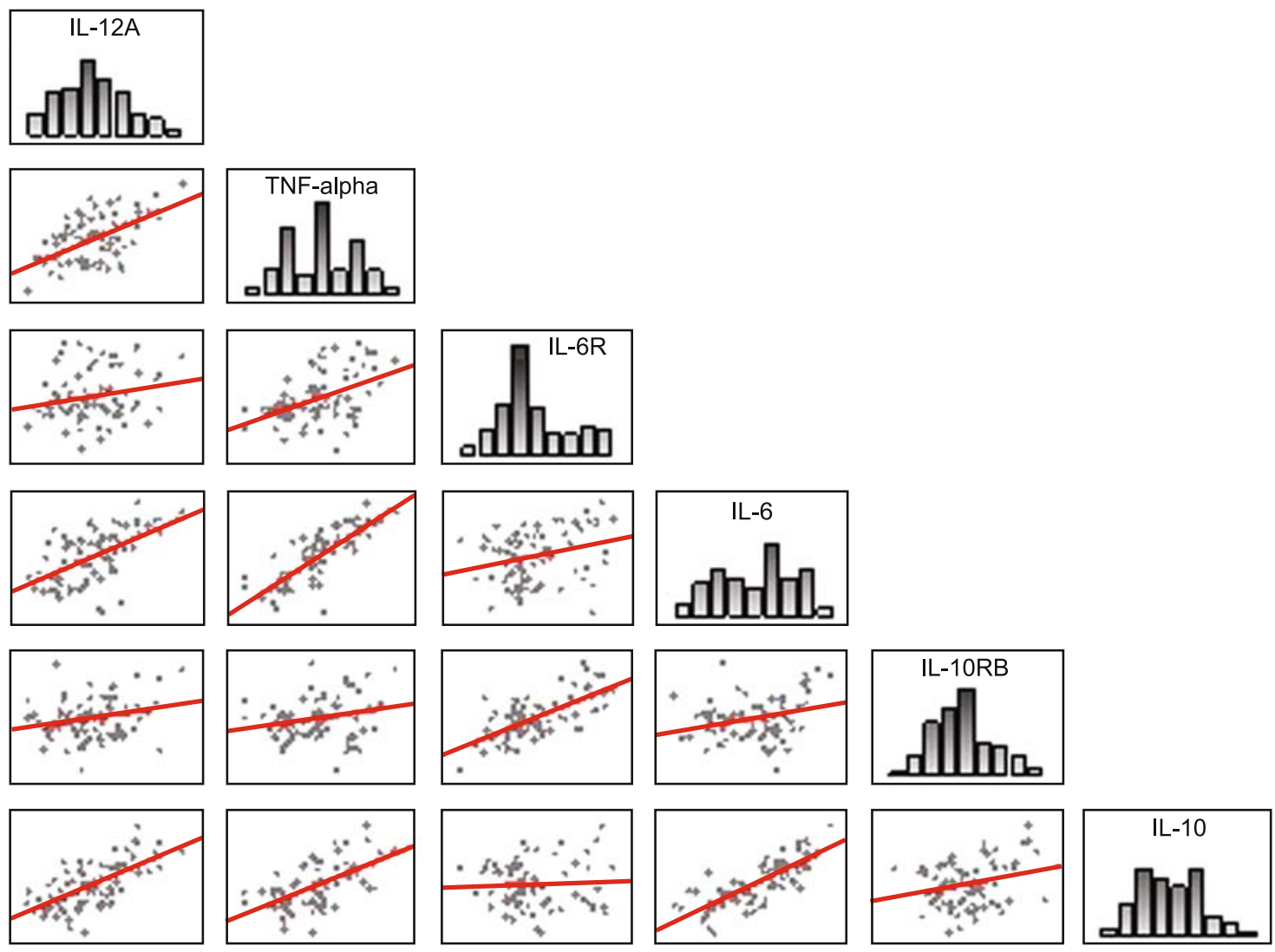

Fig. 5. Correlations among cytokines. 
it is obvious from these data the closest correlations were show for TNF with IL-6 ( $\mathrm{p}=0.001, \mathrm{r}=0.8143)$, IL-6R with IL-10RB ( $\mathrm{p}=$ $0.0001, r=0.6705)$ and IL-6 with IL-10 $(p=0.0001, r=0.7837)$. It is not possible to conclude that one of them is a cause and the second one is a consequence.

Results suggest cell differentiation into pro-inflammatory phenotype. In the future it is therefore necessary to study these populations in detail and clearly confirme the phenotype.

\section{Conclusion}

Expression of pro-inflammatory cytokines was lower in macrophages with supernatant ( $\mathrm{M} \Phi+$ sup) compared to positive control (M $\Phi+\mathrm{LPS})$. Effects of the supernatant on expression of IL-6, IL-10, IL-12 and TNF- $\alpha$ was not confirmed. It could be explained by the fact that PC-3 never encountered with macrophages in their cell life cycle. This study was in vitro study and immune system did not attack cancer cells and did not interact with them. Likely cancer cells without mutual physical contact with macrophages did not exclude substance for M2/M1 switch. There was no change in phenotype of macrophages due to PC-3 supernatant. The present study also indicated that LPS induces phenotypic M1/ M2 switch off in macrophages. Expression of pro-inflammatory cytokines was lower in cells unstimulated by LPS (M2 phenotype) compared to cells stimulated by LPS (M1 phenotype). Thus LPS can participate in creating a suitable microenvironment for carcinogenesis by overexpresion of cytokines, which can promote tumorigenesis.

\section{References}

1. Solís-Martínez R, Cancino-Marentes M, Hernández-Flores G et al. Regulation of immunophenotype modulation of monocytes-macrophages from M1 into M2 by prostate cancer cell-culture supernatant via transcription factor STAT3. Immunol Lett 2018; 196 (2018): 140-148.

2. Ridge S, Bhattacharyya D, Dervan $\mathbf{E}$ et al. Secreted factors from metastatic prostate cancer cells stimulate mesenchymal stem cell transition to a pro-tumourigenic 'activated' state that enhances prostate cancer cell migration. Internat J Cancer 2018; 142: 2056-2067.
3. Alcaide J, San Martin R, Bethencourt F, Bouraoui Y, Berriguete G. Prostatic Specific Antigen (PSA), pro-Inflammatory Cytokines, and Prostatic Pathology (benign Prostatic Hyperplasia and Cancer). Relationship with Malignancy. Oncol Urol 2009; 62: 359-366.

4. Landskron G, De la Fuente M, Thuwajit P, Thuwajit Ch, Hermoso M. Chronic Inflammation and Cytokines in the Tumor Microenvironment. J Immunol Res 2014; 2014: 1-19.

5. Caras I, Catalin T, Lerescu L, Pitica R, Melinceanu L. Influence of Tumor Cell Culture Supernatants on Macrophage Functional Polarization: In Vitro Models of Macrophage-Tumor Environment Interaction. Tumori 2011; 97: 647-654.

6. Shih J, Yuan A, Chen J, Yang P. Tumor-Associated Macrophage: Its Role in Cancer Invasion and Metastasis. J Cancer Mol 2006; 2: 101-106.

7. Mallmann M, Schmidt S, Schultze J. Macrophages in Human Cancer: Current and Future Aspects. Atlas Genet Cytogenet Oncol Haematol 2012; 16: 765-774.

8. Sánchez-Reyes K, Bravo-Cuellar A, Hernández-Flores G, LermaDíaz J, Jave-Suárez L. Cervical Cancer Cell Supernatants Induce a Phenotypic Switch from U937-Derived Macrophage-Activated M1 State into M2-like Suppressor Phenotype with Change in Toll-like Receptor Profile. BioMed Res Internat 2014; 2014: 683-694.

9. Aung H, Schroder K, Himes S, Brion K, Zuylen W. LPS Regulates Proinflammatory Gene Expression in Macrophages by Altering Histone Deacetylase Expression. FASEB J 2006; 20: 1315-1327.

10. Blagih J, Russell G. Polarizing Macrophages through Reprogramming of Glucose Metabolism. Cell Metab 2012; 15: 793-795.

11. Vignali D, Kuchroo K. IL-12 Family Cytokines: Immunological Playmakers. Nature Immunol 2012; 13: 722-728.

12. Grivennikov, S., Florian, G., Karin, M. Immunity, Inflammation, and Cancer. Cell 2011; 140: 883-899.

13. Scheller J, Chalaris A, Schmidt-Arras D, Rose-John S. The proand Anti-Inflammatory Properties of the Cytokine Interleukin-6. Biochim Biophys Acta Mol Cell Res 2011; 1813: 878-888.

14. Lu H, Ouyang W, Huang Ch. Inflammation, a Key Event in Cancer Development. Mol Cancer Res 2006; 4: 221-233.

15. Djavan B, Eckersberger E, Espinosa G, Kramer G, Handisurya A. Complex Mechanisms in Prostatic Inflammatory Response. Eur Urol Suppl 2009; 8: 872-878. 